ume of $\sim 7 \mathrm{~cm}^{3}$. Beyond this size the water ball was limited by the diameter of the glass container, touching its inner wall and spreading on the wall by wetting.

To demonstrate that the levitation is mainly due to the 'magneto-Archimedes' buoyant force, a paramagnetic water ball $\left(\chi=+0.48 \times 10^{-6}\right.$ in SI units) containing $\mathrm{CuSO}_{4}$ was levitated by the same procedures but under a little higher oxygen pressure, $18.4 \mathrm{~atm}$. Figure 2 shows a photograph of a blue ball of paramagnetic aqueous $\mathrm{CuSO}_{4}$ solution. To the best of our knowledge, there have been no reports of levitating a paramagnetic substance stably in the atmosphere. Furthermore, it has been claimed that it should be impossible to levitate a paramagnetic substance stably because a position cannot be created in the free space where the magnetic field is maximized according to one of the Maxwell relations, $\operatorname{div} \mathbf{B}=0$. But in magneto-Archimedes levitation the substance should float at the position of the minimum field horizontally, in the same position as in diamagnetic levitation.

Yasuhiro Ikezoe $^{*}$, Noriyuki Hirota ${ }^{* \dagger}$, Jun Nakagawa ${ }^{\ddagger}$, Koichi Kitazawa ${ }^{* \dagger}$

${ }^{*}$ Department of Applied Chemistry,

Department of Superconductivity,

University of Tokyo, 7-3-1 Hongo, Bunkyo-ku,

Tokyo 113-8656, Japan

†Japan Science and Technology Corporation,

4-1-8 Honcho, Kawaguchi, 332-0012 Japan

$\ddagger T D K$ Co., Ltd, Materials Research Center,

570-2 Matsugashita, Minamihatori, Narita,

286-8588 Japan

1. Beaugnon, E. \& Tournier, R. Nature 349, 470 (1991).

2. Berry, M. V. \& Geim, A. K. Eur. J. Phys. 18, 307-313 (1997).

3. Jones, T. B. J. Appl. Phys. 50, 5057-5058 (1979).

4. Khalafalla, S. E. \& Reimers, G. W. Joint Meeting MMIJ-AIME, Tokyo, Print No. TIII e5 (1972).

\section{Nicotine metabolism} defect reduces smoking

Nicotine is the primary compound in tobacco that establishes and maintains tobacco dependence ${ }^{1}$. Most of this nicotine is metabolized to cotinine by the genetically variable enzyme CYP2A6. Here we show that individuals lacking full functional CYP2A6, who therefore have impaired nicotine metabolism, are significantly protected against becoming tobacco-dependent smokers. In addition, smokers whose nicotine metabolism is thus impaired smoke significantly fewer cigarettes than those with normal nicotine metabolism.

Approximately one-third of the global population over 15 years of age smokes, despite the association of this activity with a higher incidence of many diseases including cancers and respiratory and cardiovascular

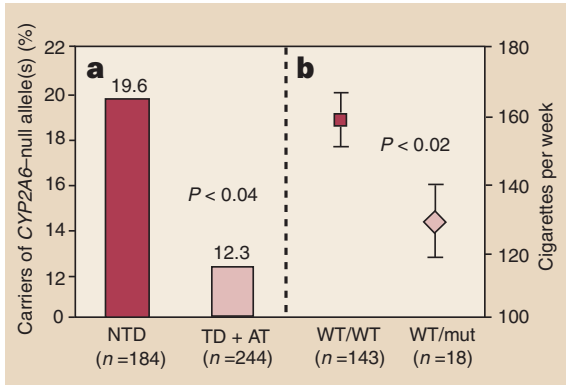

Figure 1 Effect of CYP2A6-null alleles on tobacco dependence. a, Percentage of individuals carrying CYP2A6-null alleles (1 or 2) in the never-tobaccodependent exposure control group (NTD), and the combined tobacco-dependent (TD) and alcoholand tobacco-dependent (AT) groups. b, Number of cigarettes smoked per week (mean = s.e.m.) within the TD group is lower for individuals carrying a CYP2A6-null allele. No significant difference in age between groups $(P=0.05)$; WTMT: CYP2A $6{ }^{*} 1 /{ }^{*}$; WT/mut: CYP2A6*1/*2 + CYP2A6*1/*3.

disorders. In humans, $60-80 \%$ of nicotine that results from smoking is metabolized to cotinine $^{2}$, principally by the genetically polymorphic CYP2A6 enzyme ${ }^{3,4}$. Three CYP2A6 alleles have been identified: the wild-type $\left(C Y P 2 A 6^{*} 1\right)$ and two null, or inactive, alleles $\left(C Y P 2 A 6^{*} 2\right.$ and CYP $\left.2 A 6^{*} 3\right)^{5}$.

We considered that there would be an under-representation of individuals with impaired nicotine metabolism (carriers of null CYP2A6 alleles) in a tobaccodependent population. Genotype frequencies for CYP2A6 alleles ${ }^{5}$ were quantified in three groups: a tobacco-dependent group (TD, $n=164,52 \%$ male) defined by DSMIV and Fagerstrom criteria, an alcohol- and tobacco-dependent group (AT, $n=80,84 \%$ male), and a never-tobacco-dependent exposure control group who had each tried smoking but had never become dependent (NTD, $n=184,52 \%$ male). Subjects in all groups had no other psychoactive drug dependencies (DSM-IV criteria). We found that, among all dependent smokers (TD + AT), the frequency of individuals with impaired nicotine metabolism (carriers of 1 or 2 CYP2A6-null alleles) was lower than in the control group $(12.3 \%$ versus $19.6 \%$, $P<0.04$, chi-square; odds ratio $=1.74,95 \%$ confidence intervals 1.02-2.94; Fig. 1a). These data show that impaired nicotine metabolism protects a smoker against becoming dependent: in fact, even a single CYP2A6-null allele (that is, heterozygosity) is sufficient to significantly reduce the risk of tobacco-dependence.

Dependent smokers adjust their smoking to maintain constant blood and brain nicotine concentrations ${ }^{6,7}$. Therefore we tested whether dependent smokers with impaired nicotine metabolism would need to smoke fewer cigarettes. Within the TD group, those heterozygous for CYP2A6-null alleles smoked significantly fewer cigarettes per week than smokers with two CYP2A6 active alleles (129 versus 159 cigarettes per week; $t$-test $P<0.02$; Fig. 1b). These data suggest that CYP2A6-mediated nicotine metabolism is a significant determinant of the number of cigarettes consumed by dependent smokers and that heterozygosity in a single gene, the CYP2A6 gene, significantly decreases this complex drug-taking behaviour.

Individuals carrying CYP2A6-null alleles may have a decreased risk of developing tobacco-related cancers ${ }^{8}$ and other medical complications because they have a decreased risk of becoming a smoker and, if they do become dependent, they smoke less than those without impaired nicotine metabolism. As tobacco smoke contains nitrosamines which can be activated to carcinogens by CYP2A6 (refs 9,10), individuals who carry CYP2A6-null alleles may also be less efficient at activating tobacco-smoke procarcinogens to carcinogens. These three factors may explain why there could be a reduction in tobacco related cancers for carriers of CYP2A6null alleles.

CYP2A6 genotype may significantly affect nicotine levels from nicotine sources other than cigarettes, such as nicotinereplacement therapies (for example, patch, gum, spray). This may be of growing importance as such therapies are used increasingly for long-term maintenance against tobacco dependence and for treatment of other syndromes (such as Alzheimer's disease, Tourette's syndrome, ulcerative colitis). In addition, the protective effect of CYP2A6-null alleles against the risk of becoming tobacco-dependent and in decreasing consumption suggests that inhibiting this enzyme may be a new way to help prevent and treat tobacco smoking.

Michael L. Pianezza, Edward M. Sellers, Rachel F. Tyndale

Addiction Research Foundation of Ontario, Centre for Research in Women's Health, Department of Pharmacology, Medicine and Psychiatry, University of Toronto, Toronto, M5S 1A8, Canada

e-mail: r.tyndale@utoronto.ca

1. Henningfield, J. E., Miyasato, K. \& Jasinki, D. R. J. Pharmacol. Exp. Ther. 234, 1-12 (1985).

2. Benowitz, N. L. et al. J. Pharmacol. Exp. Ther. 268, 296-303 (1994).

3. Nakajima, M. et al. Drug Met. Disp. 24, 1212-1217 (1996).

4. Messina, E., Tyndale, R. F., \& Sellers, E. M. J. Pharmacol. Exp. Ther. 282, 1608-1614 (1997)

5. Fernandez-Salguero, P. et al. Am. J. Hum. Genet. 57, 651-660 (1995).

6. McMorrow, M. J. \& Foxx, R. M. Psychol. Bull. 93, 302-327 (1983).

7. Russel, M. S. H. in Advances in Behavioural Biology (eds Martin, W. R., Vauhon, G. R., Iwamoto, E. T., \& David, L.) 25-50 (Plenum, New York, 1987).

8. Law, M. R., Morris, J. K., Watt, H. C., \& Wald, N. J. Brit. J. Cancer 75, 1690-1693 (1997).

9. Crespi, C.L. et al. Carcinogenesis 11, 1293-1300 (1990). 10. Yamazaki, H. et al. Carcinogenesis 13, 1789-1794 (1992). 